\title{
Search for exotic neutrino interactions by XMASS-I detector
}

\section{Hiroshi Ogawa ${ }^{a, *}$ on behalf of the XMASS Collaboration}

(a complete list of authors can be found at the end of the proceedings)

${ }^{a}$ College of Science and Technology, Nihon University

1-8-14 Kanda Surugadai, Chiyoda-ku, Tokyo, Japan

E-mail: ogawa.hiroshi@nihon-u.ac.jp

XMASS is a multi-purpose experiment using liquid xenon (LXe) and is located at the Kamioka Observatory in Japan. The detector consists is single-phase and has a $832 \mathrm{~kg}$ active volume, a low energy threshold, low background and large target mass. With XMASS, it is possible to also explore low energy neutrino physics to search for beyond teh standard model (SM) physics. Here we present a search for exotic neutrino-electron interactions that could be produced by a neutrino millicharge, a neutrino magnetic moment, or dark photons, using solar neutrinos. We analyzed data taken between November 2013 and March 2016, giving us a 711days dataset. No significant signal was observed above the predicted background in the detector. We thus obtained an upper limit for neutrino millicharge of $5.4 \times 10^{-11} \mathrm{e}$ for all flavors of neutrino. We also constrainted individual flavors to be smaller than $7.3 \times 10^{-12} e$ for $v_{e}, 1.1 \times 10^{-11} e$ for $v_{\mu}$, and $1.1 \times 10^{-11} e$ for $v_{\tau}$. These limits for $v_{\mu}$ and $v_{\tau}$ are currently the best direct experimental limits. We also obtain an upper limit for the neutrino magnetic moment of $1.8 \times 10^{-10} \mu_{B}$. In addition, we obtain upper limits for the coupling constant of dark photons in the $U(1)_{B-L}$ model of $1.3 \times 10^{-6}$ if the dark photon mass is $1 \times 10^{-3} \mathrm{MeV} / c^{2}$, and $8.8 \times 10^{-5}$ if it is $10 \mathrm{MeV} / c^{2}$. In particular, we almost exclude the possibility to understand the muon $g-2$ anomaly as originating from dark photons.

$37^{\text {th }}$ International Cosmic Ray Conference (ICRC 2021)

July 12th-23rd, 2021

Online - Berlin, Germany

\footnotetext{
*Presenter
} 


\section{Intorduction}

In recent years, the larger size and lower background of dark matter search detectors have opened up a new frontier of searching for new physics other than dark matter search for these detectors. One of these areas is the search for exotic interactions by neutrinos from the Sun. Solar neutrinos have been observed by various detectors, but pp-chain neutrinos, which have the highest flux, have not yet been observed in neutrino-electron interactions due to their low energy. When observing pp-chain neutrinos with a current dark matter detector, for example, the XMASS-I detector, the background events is required to reduce to $1 / 100$ or less.

However, if there is an exotic neutrino interaction not foreseen in the standard model, it is expected that the number of events will increase, especially in the low energy region. This opens the door for observations with low-threshold dark matter search detectors. In this paper, we search for the exotic interaction of neutrinos from the Sun with the XMASS dark matter detector [1]. Here we investigate new neutrino properties such as neutirno magnetic moment, millicharge, and dark photon-mediated interactions.

In the Standard Model (SM), the electric charge of neutrinos is assumed to be zero. But neutrinos may have a slight charge, as charge quantization has not been proven [2]. Also some extensions of the SM yield neutrino magnetic moments at currently observable levels, which are of $O\left(10^{-12} \sim 10^{10}\right) \mu_{B}$ [3]. In both cases, an electro-magnetic term has to be added in the cross section of neutrino-electron interaction. Another scenario for exotic interactions is the possibility that a dark photon mediates a neutrino interaction with electrons. Here we investigate dark photons derived from a gauged $U(1)_{B-L}$ with the mass $M_{A^{\prime}}$ and the coupling constant $g_{B-L}$ [4].

Our detector simulation translates theoretical cross sections for these exotic interactions into spectra of deposited energy in the detector. Figure 1 shows these deposited energy spectra for each of the neutrino interactions in xenon we investigate. Our analysis considers atomic effects in xenon that affect the signal distribution. These appear as steps in these energy distributions at atomic binding energies. To model these atomic effects, the relative random phase approximation (RRPA) [5] is used in millicharge analysis, and the free electron approximation (FEA) [6] is used in the magnetic moment and dark photon analysis. The event rate due to a neutrino millicharge and magnetic moment are proportional to the second power of these quantities while the event rate for dark photons is proportional to the forth power of $g_{B-L}$ with a dependence on $M_{A^{\prime}}$.

\section{The XMASS detector}

XMASS [7] is a multi-purpose detector with an ultra pure liquid xenon (LXe) target, designed for single-phase readout. Its simple structure is easily scalable and optimized for high ligh collection. Xenon's high atomic number $(\mathrm{Z}=54)$ and high density $(\sim 2.96 \mathrm{~g} / \mathrm{cc})$ allow a compact detector design that is self-shielding against external gammas, which will be absorbed within a short distance from the detector's inner surface.

The XMASS detector [8] was constructed at the Kamioka Laboratory under an overburden of 2700 m.w.e. A cylindrical water tank of $10 \mathrm{~m}$ diameter and $11 \mathrm{~m}$ height contains 72 20-inch PMTs and ultra pure water. It constitutes a water-Cherenkov outer detector (OD) and serves as a shield against fast neutrons and external gammas as well as an active muon veto. The inner detector uses a 


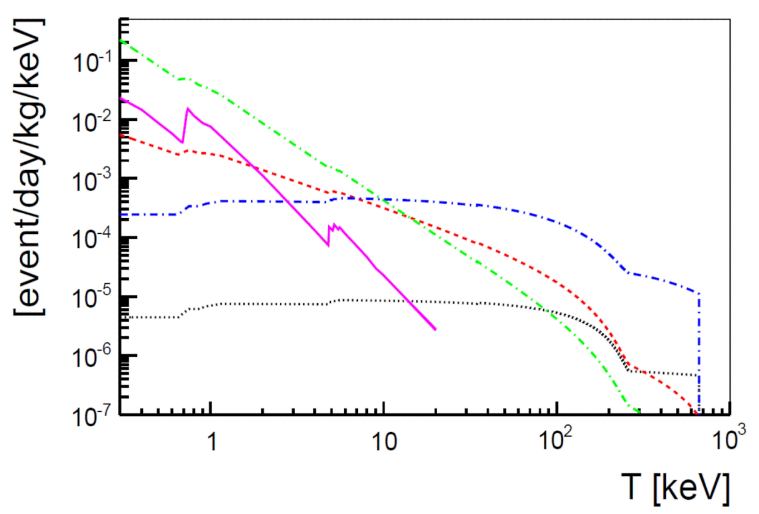

Figure 1: The deposited energy spectra for exotic neutrino interactions with electron in xenon. The magentasolid line shows a model where the neutrino has a millicharge $\left(1.5 \times 10^{-12} e\right)$. The red-dashed line shows a model where the neutrino has a magnetic moment $\left(1 \times 10^{-10} \mu_{B}\right)$ The green-dash-dotted and blue-dash-dotted line show models where neutrino interacts with electrons through dark photons with $g_{B-L}=1 \times 10^{-6}$ and $M_{A^{\prime}}=1 \times 10^{-3} \mathrm{MeV} / c^{2}$ and with $g_{B-L}=1 \times 10^{-4}$ and $M_{A^{\prime}}=10 \mathrm{MeV} / c^{2}$, respectively. The black-dotted line shows the Standard Model neutrino-electron weak interaction.

total of $1107 \mathrm{~kg}$ of liquid xenon, of which $832 \mathrm{~kg}$ serve as an active scintillator target in its sensitive inner volume.

642 photomultiplier tubes (PMTs, Hamamatsu R10789-11 and Hamamatsu R10789-11MOD) are mounted on the pentakis-dodecahedral inner surface of the detector at radii slightly larger than but close to $40 \mathrm{~cm}$. The photo-cathode coverage of that inner surface is about $62.4 \%$. The quantum efficiency of the PMTs at the liquid xenon scintillation wavelength $(\sim 175 \mathrm{~nm})$ is about $30 \%$ on average.

The detector construction of XMASS-I was completed in September 2010. Physics data were taken between December 2010 and May 2012 in the commissioning phase. After a detector refurblishiment [9] that significantly reduced the background in the detector, we succeeded in acquiring data continuously for more than 5 years from November 2013 onward. During this period, the scintillation light yield response was traced by externally applying a ${ }^{60} \mathrm{Co}$ source in the OD water just outside of the inner detector's cryostat, and inserting a ${ }^{57} \mathrm{Co}$ source into the inner detector every one or two weeks. Data taking with the XMASS-I detector was completed in February 2019.

\section{Analysis}

We analyzed the data accumulated between Novermber 2013 and March 2016. The total analyzed livetime is 711 days. Event selection is shown below. First, among the events that do not have an OD trigger, the afterpulse events of ID PMTs and the Cherenkov events generated by the interaction of $\beta$-rays generated from ${ }^{40} \mathrm{~K}$ mainly in the PMT photocathodes under the quartz window of the PMTs are removed. Then, background events originating from the surface of the detector are removed by performing an effective volume cut by based on a timing based positional 
event reconstruction ( $\mathrm{R}$ (timing) $<38 \mathrm{~cm}$ ) and another, $\mathrm{PE}$ distribution based reconstruction $(\mathrm{R}$ $(\mathrm{PE})<20 \mathrm{~cm}$ ). The weight of the xenon in this effective volume is approximately $97 \mathrm{~kg}$.

These selection criteria were applied to both signal MC and background MC as well as to the data. We performed detector simulation using the Geant 4 simulation package. For the signal MC, event selection was performed by weighting the electron event $\mathrm{MC}$, which was produced uniform in the detector, with the energy deposit spectrum calculated for the respective interaction shown in Fig 1. The bottom of Fig 3 shows the signal efficiency for the millichagre signal. At the same time, the scintillation efficiency of liquid xenon and the systematic error introduced by the event selection were evaluated.

Background MC is generated for each detector material and radioactive isotopes (RIs) with the XMASS MC [10]. The same event selection is applied, produced with the same statistics as the data. Fig 2 shows the energy distribution of the BG simulation from 2-200 keV after event selections. The dominant $\mathrm{BG}$ component for $\mathrm{E}<30 \mathrm{keV}$ derives from RIs at the inner surface of the detector. These RIs are ${ }^{238} \mathrm{U},{ }^{235} \mathrm{U},{ }^{232} \mathrm{Th},{ }^{40} \mathrm{~K},{ }^{60} \mathrm{Co}$ and ${ }^{210} \mathrm{~Pb}$ present in the PMTs and ${ }^{210} \mathrm{~Pb}$ in the copper used in the detector structure holding the PMTs. All detector materials except for the LXe had been assayed using high-purity germanium detectors or a surface alpha-ray counter [11]. Some of these RI events remain after event selection by being accidentally mis-reconstructed within the fiducial volume in the position reconstruction which is why we call them mis-reconstruction events. For these mis-reconstruction events, we applied a data-driven correction to the simulated BG spectrum in order to take into account the systematic difference in the mis-reconstruction rate near dead PMTs ( 9 out of 642 PMTs which had been found to noisy or delivered strange response and had been turned off). The systematic error evaluation focussed on the detector's inner surface condition and this correction for mis-reconstruction near dead ID PMTs for energies $\mathrm{E}<30 \mathrm{keV}$. For $\mathrm{E}>30 \mathrm{keV}$, RI dissolved in the LXe become the dominant BG component. Impurities in LXe such as ${ }^{222} \mathrm{Rn},{ }^{85} \mathrm{Kr},{ }^{39} \mathrm{Ar}$ and ${ }^{14} \mathrm{C}$ were evaluated [12]. Also xenon isotopes generated by neutrons such as ${ }^{131 m} \mathrm{Xe},{ }^{133} \mathrm{Xe},{ }^{125} \mathrm{I}$ and ${ }^{136} \mathrm{Xe}$ were found to be produced in the cooling towers for the LXe, which were outside of the water shield.

Signal MC and Background MC follow the changes in the optical parameter of the liquid xenon as measured by our regular calibration data throughout the whole data-taking period.

\section{Results}

$\chi^{2}$ fits were performed on the data using BG MC with its proper systematic uncertainties and the respective signal MC for the respective interaction model. The energy range was set to be 2-15 $\mathrm{keV}$ for the neutrino millicharge and $2-200 \mathrm{keV}$ for the neutrino magnetic moment and dark photon searches to fully cover the respective signal regions. We note that the constraints due to ${ }^{14} \mathrm{C},{ }^{39} \mathrm{Ar}$, ${ }^{131 m} \mathrm{Xe}$ and ${ }^{133} \mathrm{Xe}$ are not applied in the dark photon or neutrino magnetic moment analysis becasue the spectrum of expected signals and these BGs are overlapped at energies.

Fig. 3 shows the data amd best-fit signal + background MC with the signal MC at the $90 \%$ confidence level (CL) upper limit for the neutrino millicharge analysis. We found no significant signal excess for any of our signal models. The upper limit for neutrino millicharge at $90 \% \mathrm{CL}$ is though for the $5.4 \times 10^{-12} e$ if all three species fo neutrino have a common millicharge. For the neutrino (not anti-neutrino) case, our limit is more stringent than the previous limit in [13]. The 


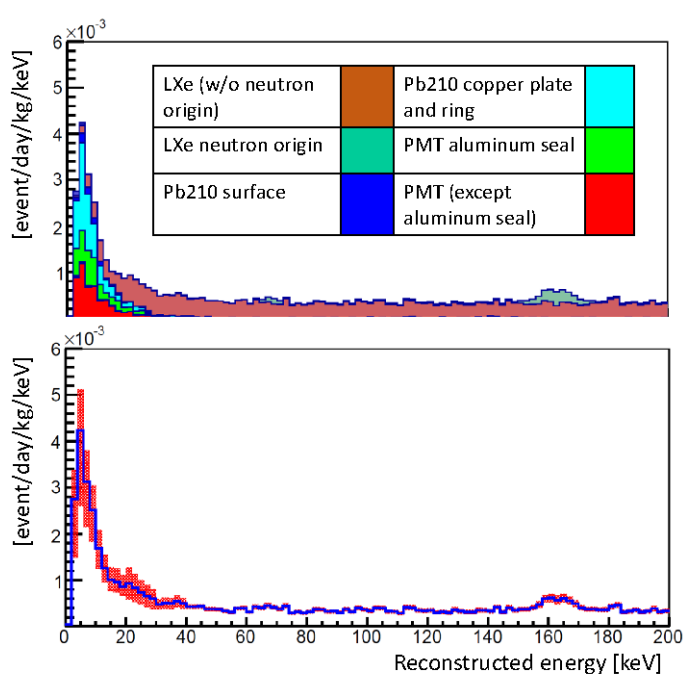

Figure 2: (Top) The energy distribution of the BG simulation after event selection from 2 to $200 \mathrm{keV}$. The contributions to the $\mathrm{BG}$ originating from various types of events are indicated by the colored histograms in the top panel. (Bottom) The cumulative contribution of all the systematic errors is indicated by the red band in the bottom panel.

upper limit for each flavor of neutrino produced by neutrino oscillations of solar neutrinos was calculated to $7.3 \times 10^{-12} e$ for $v_{e}, 1.1 \times 10^{-11} e$ for $v_{\mu}$, and $1.1 \times 10^{-11} e$ for $v_{\tau}$ and the strongest limit was given for positive neutrinos.

We also searched for signal excesses due to a neutrino magnetic moment and dark photons, but again found no significant excess. Fig. 4 shows the energy distribution of the data and the best-fit signal BG with the signal MC at the $90 \% \mathrm{CL}$ upper limit for a neutrino magnetic moment (top) and dark photons (middle and bottom). The best fit neutrino magnetic moment was $\mu_{v}=$ $1.3 \times 10^{-10} \mu_{B}$ and the $90 \% \mathrm{CL}$ upper limit is $1.8 \times 10^{-10} \mu_{B}$. We investigated dark photon masses from $1 \times 10^{-3} \mathrm{MeV} / \mathrm{c}^{2}$ to $1 \times 10^{3} \mathrm{MeV} / \mathrm{c}^{2}$ of $M_{A^{\prime}}$ of dark photon. The middle and bottom figures of Fig. 4 are the results when $M_{A^{\prime}}=1 \times 10^{-3} \mathrm{MeV} / \mathrm{c}^{2}$ and $M_{A^{\prime}}=10 \mathrm{MeV} / \mathrm{c}^{2}$, respectively. The upper limits for $\mathrm{g}_{B-L}$ with $\mathrm{M}_{A^{\prime}}=1 \times 10^{-3} \mathrm{MeV} / \mathrm{c}^{2}$ and $M_{A^{\prime}}=10 \mathrm{MeV} / \mathrm{c}^{2}$ are $1.3 \times 10^{-6}$ and $8.8 \times 10^{-5}$ at $90 \% \mathrm{CL}$, respectively. The $90 \%$ exclusion limit of our analysis is shown in Fig 5 with together with other existing exclusion limits and allowed regions. Most of the parameter space for $(g-2)$ dark photon prediction was excluded by our analysis.

\section{Conclusions}

We have searched for exotic neutrino-electron interactions that could be produced by a neutrino millicharge, by a neutrino magnetic moment, or by dark photons using solar neutrinos in XMASS. We analyzed the data between November 2013 and March 2016 which corresponds to 711days live days. No significant signals have been observed over the predicted background in detector. We obtained an upper limit for neutrino millicharge of $5.4 \times 10^{-11} \mathrm{e}$ for all flavors of neutrino. We also derive limits for individual flavors that are $7.3 \times 10^{-12} e$ for $v_{e}, 1.1 \times 10^{-11} e$ for $v_{\mu}$, and $1.1 \times 10^{-11} e$ 
for $v_{\tau}$. The limits for $v_{\mu}$ and $v_{\tau}$ are the best limits from direct detection. We also obtain an upper limit for the neutrino magnetic moment of $1.8 \times 10^{-10} \mu_{B}$. Finally, we obtain an upper limits for the coupling constant of dark photons in the $U(1)_{B-L}$ model of $1.3 \times 10^{-6}$ if the dark photon mass is $1 \times 10^{-3} \mathrm{MeV} / c^{2}$, and $8.8 \times 10^{-5}$ if the mass is $10 \mathrm{MeV} / c^{2}$. This limit almost excludes the possibility to understand the muon $g-2$ anomaly by dark photons.

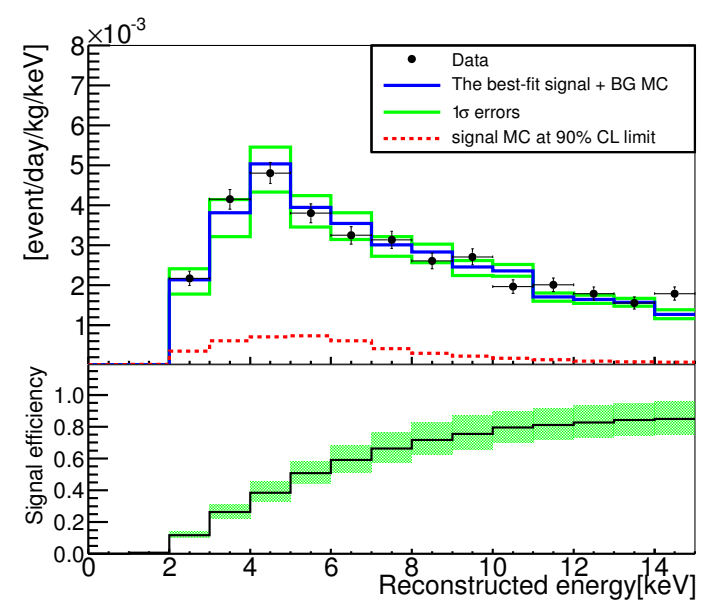

Figure 3: (Top) The energy distribution after applying all cuts. The black points show the data. The blue histograms show the best-fit signal + BG MC simulation with $1 \sigma$ errors shown by the green histograms. The red-dotted histograms show the 90\% CL upper limit for the neutrino-millicharge signal. (Bottom) Signal efficiency curve for the millicharge analysis.

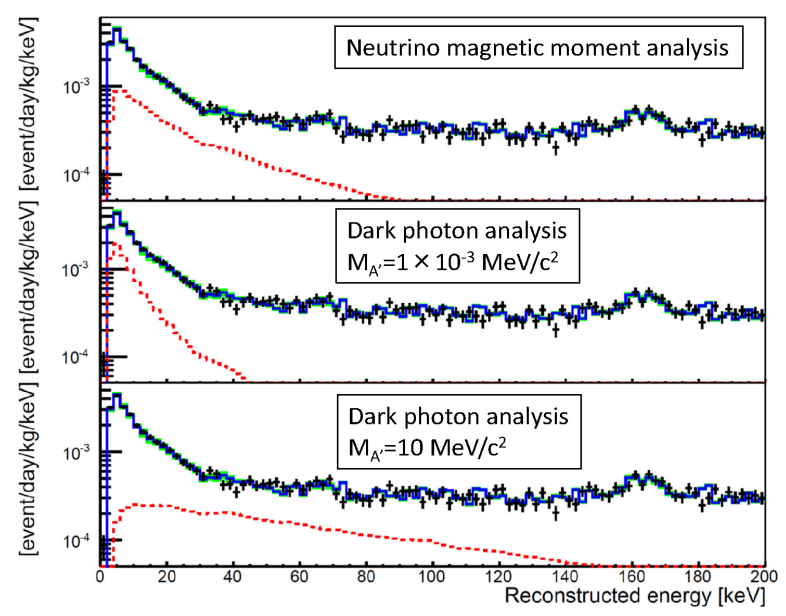

Figure 4: The energy distribution of the data, the best fit signal + BG and the 90\% CL signal limit from 2 to $200 \mathrm{keV}$ for the neutrino magnetic moment analysis (top) and the dark photon analysis (middle: dark photon mass $M_{A^{\prime}}=1 \times 10^{-3} \mathrm{MeV} / c^{2}$, bottom $M_{A^{\prime}}=10 \mathrm{MeV} / c^{2}$ ). The black points show the data. The blue histogram shows the signal + BG MC for the best fit with $1 \sigma$ errors shown by the green histograms. The red-dotted histogram shows the $90 \% \mathrm{CL}$ upper limit for the signal. 


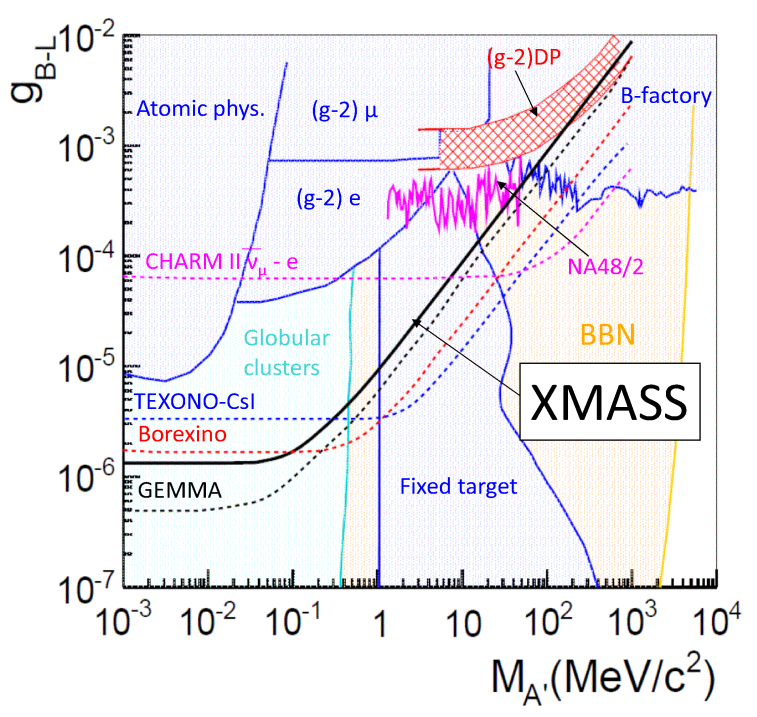

Figure 5: $90 \% \mathrm{CL}$ exclusion limits and allowed region on the coupling constant $g_{B-L}$ as a function of the dark photon mass $M_{A^{\prime}}$. The black-solid line shows the exclusion limit of our analysis (XMASS). The $2 \sigma$-allowed-region band from the muon $(g-2)$ experiment is shown as " $(g-2)$ DP" as the red-meshed region. The blue and magenta regions are excluded by laboratory experiments respectively. The cyan and orange regions are excluded by cosmological and astrophysical constraints respectively. The dotted lines are the estimated limit curves from neutrino-scattering experiments (GEMMA $\left(\bar{v}_{e}\right)$, Borexino (solar $v$ ), TEXONO-CsI $\left(\bar{v}_{e}\right)$ and CHARM II $\left.\left(\bar{v}_{\mu}\right)\right)$. These exclusion regions and curves are summarized in [4].

\section{Acknowledgents}

We gratefully acknowledge the cooperation of the Kamioka Mining and Smelting Company. This work was supported by the Japanese Ministry of Education, Culture, Sports, Science and Technology, the joint research program of the Institute for Cosmic Ray Research (ICRR), the University of Tokyo, Grant-in-Aid for Scientific Research, JSPS KAKENHI Grant No. 19GS0204, 26104004, and 19H05805 and partially by the National Research Foundation of Korea Grant funded by the Korean Government (NRF-2011-220-C00006).

\section{References}

[1] K. Abe et al., Phys. Lett. B 809 (2020) 135741.

[2] K. Kouzakov and A. Studenikin, Phys. Rev. D 95 (2017) 055013.

[3] M.B. Voloshin et al., JETP 64 (1986) 446.

[4] S. Bilmis et al.. Phys.Rev. D 92 (2015) 033009.

[5] J-W. Chen et al., Phys. Lett. B 774 (2017) 656.

[6] V. Kopeikin et al., Phys. At. Nucl. 60 (1997) 1859

[7] Y. Suzuki, arXiv:hep-ph/0008296. 
[8] K. Abe et al., Nucl. Instr. and Meth. A 716 (2013) 78.

[9] H.Ogawa, AIP Conf.Proc. 1549 (2013) 90-93.

[10] K. Abe et al., Phys. Lett. B 789 (2019) 45.

[11] K. Abe, et al., Nucl. Instr. and Meth. A884 (2018) 157.

[12] K. Abe et al., Prog. Theor. Exp. Phys. 2018 (2018) 053D03.

[13] F. Della Valle et al., Euro. Phys. J. C76 (2016) 24.

\section{Full Authors List: XMASS Collaboration}

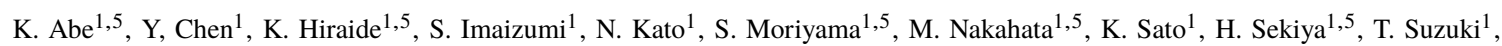

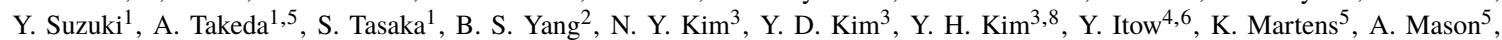
M. Yamashita ${ }^{5}$, K. Miuchi ${ }^{7}$, Y. Takeuchi ${ }^{7,5}$, K. B. Lee $^{8}$, M. K. Lee ${ }^{8}$, Y. Fukuda ${ }^{9}$, H. Ogawa ${ }^{10}$, K. Ichimura ${ }^{11,5}$, Y. Kishimoto ${ }^{11,5}$, K. Nishijima ${ }^{12}$, K. Fushimi $^{13}$, B. D. Xu ${ }^{14,5}$, K. Kobayashi ${ }^{15,5}$, S. Nakamura ${ }^{16}$

${ }^{1}$ Kamioka Observatory, Institute for Cosmic Ray Research, the University of Tokyo, Higashi-Mozumi, Kamioka, Hida, Gifu 5061205, Japan

${ }^{2}$ Center for Axion and Precision Physics Research, Institute for Basic Science, Daejeon 34051, South Korea

${ }^{3}$ Center for Underground Physics, Institute for Basic Science, 70 Yuseong-daero 1689-gil, Yuseong-gu, Daejeon 305-811, South Korea

${ }^{4}$ Institute for Space-Earth Environmental Research, Nagoya University, Nagoya, Aichi 464-8601, Japan

${ }^{5}$ Kavli Institute for the Physics and Mathematics of the Universe (WPI), the University of Tokyo, Kashiwa, Chiba 277-8582, Japan

${ }^{6}$ Kobayashi-Maskawa Institute for the Origin of Particles and the Universe, Nagoya University, Furo-cho, Chikusa-ku, Nagoya, Aichi 464-8602, Japan

${ }^{7}$ Department of Physics, Kobe University, Kobe, Hyogo 657-8501, Japan

${ }^{8}$ Korea Research Institute of Standards and Science, Daejeon 305-340, South Korea

${ }^{9}$ Department of Physics, Miyagi University of Education, Sendai, Miyagi 980-0845, Japan

${ }^{10}$ Department of Physics, College of Science and Technology, Nihon University, Kanda, Chiyoda-ku, Tokyo 101-8308, Japan

${ }^{11}$ Research Center for Neutrino Scientce, Tohoku Univeristy, Sendai 980-8578, Japan

${ }^{12}$ Department of Physics, Tokai University, Hiratsuka, Kanagawa 259-1292, Japan

${ }^{13}$ Department of Physics, Tokushima University, 2-1 Minami Josanjimacho Tokushima city, Tokushima 770-8506, Japan

${ }^{14}$ Department of Engineering Physics, Tsinghua University, Haidian District, Beijing, China 100084

${ }^{15}$ Waseda Research Institute for Science and Engineering, Waseda University, 3-4-1 Okubo, Shinjuku, Tokyo 169-8555, Japan

${ }^{16}$ Department of Physics, Faculty of Engineering, Yokohama National University, Yokohama, Kanagawa 240-8501, Japan 